Nabil B. AMRANI ${ }^{1,2}$, Laurent SAINTIS ${ }^{1}$, Driss SARSRI ${ }^{2}$, Mihaela BARREAU ${ }^{2}$

${ }^{1}$ LARIS, Polytech Angers, University of Angers, France

${ }^{2}$ LTI, National School of Applied Sciences, ENSA-Tangier, UAE, Morocco

\title{
EVIDENTIAL NETWORKS FOR EVALUATING PREDICTIVE RELIABILITY OF MECHATRONICS SYSTEMS UNDER EPISTEMIC UNCERTAINTIES
}

\begin{abstract}
In reliability predicting field, the probabilistic approaches are based on data relating to the components which can be precisely known and validated by the return of experience REX, but in the case of complex systems with high-reliability precision such as mechatronic systems, uncertainties are inevitable and must be considered in order to predict with a degree of confidence the evaluated reliability. In this paper, firstly we present a brief review of the non-probabilistic approaches. Thereafter we present our methodology for assessing the reliability of the mechatronic system by taking into account the epistemic uncertainties (uncertainties in the reliability model and uncertainties in the reliability parameters) considered as a dynamic hybrid system and characterized by the existence of multi-domain interaction between its failed components. The key point in this study is to use an Evidential Network "EN" based on belief functions and the dynamic Bayesian network. Finally, an application is developed to illustrate the interest of the proposed methodology. Keywords: epistemic uncertainties, uncertainty of model, belief function, evidential network, reliability, mechatronics, multi-domain interactions
\end{abstract}




\section{Introduction and context}

The uncertainty modelling is one of the most challenging problems in reliability studies of mechatronic systems [1], the Return of experience REX is insufficient to validate the failure rates or the probability of failure laws accurately. The ideal is to have a sufficient amount of information about the system failure in order to accurately estimate the failure rate and improve the level of confidence, by taking into account the epistemic uncertainties.

The goal in this paper is to predict the impreciseness of reliability of mechatronic systems by taking into account the epistemic uncertainties (Uncertainty of model and parameters) and simultaneously modelling the uncertainties related to multi-domain interactions.

Firstly, we present a relatively detailed presentation of some studies which are very close to the issue of epistemic uncertainties in reliability analysis, then we propose our contribution based on Evidential Network "EN". Then, we present a brief definition of belief function Belief functions "BEL" and Plausibility "PL"), and then their implementation in reliability assessment.

Uncertainties can be classified into two main categories [2, 3], as presented in fig. 1.

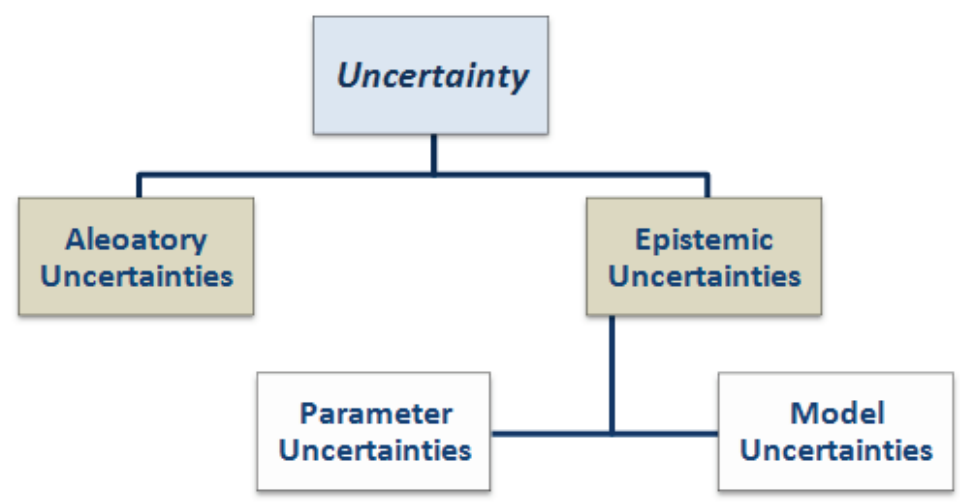

Fig. 1. Types of uncertainties

The epistemic uncertainties result from imprecise, unobservable, cognitive and reducible knowledge. Epistemic uncertainty derives from some level of ignorance, or incomplete information, of the system or the surrounding environment. Epistemic uncertainties have been classified into two types: Model and parameter uncertainties. 
Model uncertainties are related to the structure of the system, meaning an imprecision at the level of logical relations between the components, and representing the common modes of failure. Parameter uncertainties represent the lack of information for the input data of reliability model, such as components reliability data (failure rate, repair rate) or data related to the operating profile, the uncertainties of model [4] system are divided in two cases: There is complete independence between component failures. The system fails according to its structure function. There is a complete dependence between component failures. The system fails whenever a component fails; it behaves as a series system.

The random uncertainties are characterized by variability, stochastic, and irreducible aspects, and random events, such as the event initiator, failure at solicitation or failure in functioning, for which we associate Poisson models, binomial, et cetera.

Several researchers in this framework $[4,5,6,7,8,10]$ have addressed to model epistemic uncertainties in reliability analysis. The date of failure cannot be predicted precisely. However, with a given degree of confidence or belief; many other authors treat the problem of precision with probabilities densities and subjective probability in the form of expert knowledge. Also, some approaches [8, $9,10,11]$ showed that a priori probability or a logical analysis could be used with an empirical probability, envelope of probabilities, imprecise probabilities [13], fuzzy numbers or belief functions to overcome epistemic uncertainty. You and Tonon [17] applied imprecise probability methods to event-tree analysis. Simon and Weber $[6,14,15]$ present an approach based on belief function to treat uncertainties during reliability analysis as classical systems, bridge structures, and hydraulic systems. The final results are expressed with interval bounds, which indicate a relatively high uncertainty for making a final decision.

Later on, Duy [16] used belief functions in the form of p-boxes along with Monte-Carlo simulations to model propagation of uncertainty. Zhong[18] develops an approach based on Bayesian networks in order to analyze the uncertainty of reliability parameters for mechatronic systems, and a continuous DBN model is carried out by a non-parametric belief propagation (NBP) algorithm. This method was tested on the Active suspension system for a vehicle.

An overview of these approaches, which is presented in M. Sallak [4] and Utkin [2], concludes that the belief network (Evidentials Networks "ENs") has an excellent results in modelling uncertainties. 


\section{Contribution: Evidential networks for mechatronic systems}

\subsection{Global Methodology}

We propose in this paper an original methodology for evaluating predictive reliability of interactive mechatronic systems by taking into account the epistemic uncertainties in the design phase, as described in fig. 2.

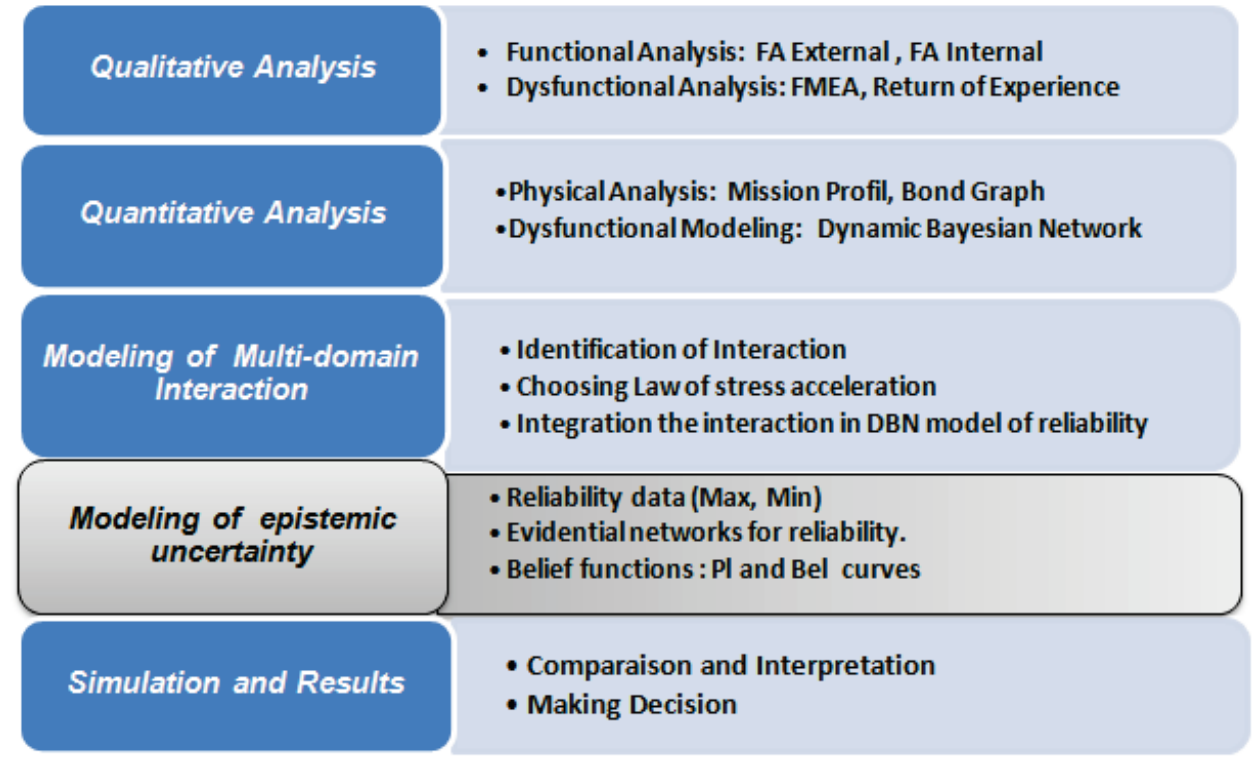

Fig. 2. Global approach for the predictive evaluation of mechatronic systems

In our recent work [19], we proposed a methodology for evaluating the predictive reliability of the mechatronic system by taking into account the multidomain interaction, all these phases (Qualitative Analysis, Quantitative Analysis, Modelling of multi-domain interaction) are developed. The new contribution in this paper is the modeling of epistemic uncertainties to predict with precision the reliability of the mechatronic system.

Here, we propose a particular Dynamic Evidential networks to handle the epistemic uncertainty problem for mechatronic systems with multi-domain interactions. 


\section{Evidential Networks in reliability for mechatronic system}

\subsection{Belief functions}

Belief functions are a mathematical tool treating the random nature of information and the imprecise information [13]. The information is represented as masses (probability weights) assigned to intervals. Belief functions theory is a unique framework for representing and manipulating random and epistemic uncertainties. Let us consider a discrete random variable $\mathrm{x}$ taking values in the frame of discernment, where all of its possible events are mutually exclusive elementary propositions. $X=\left(x_{1} ; \ldots n n\right), x$ can also be seen as a question, and $\Omega$ as the set of possible answers to that question. A mass function $m$ that assigns values to these sets in the interval $[0 ; 1]$, such that:

$$
\sum_{A \subset \Omega} m(A)=1
$$

where $\mathrm{m}(\mathrm{A})$ represents the part of belief assigned to the hypothesis that the truth is in the subset A, without further dividing this belief to a strict subset of A. Two other important functions to represent knowledge are defined: the Belief function (Bel) and the Plausibility function (Pl). They are presented by the following expressions:

$$
\begin{aligned}
\operatorname{Bel}(A)=\sum_{\varphi \neq A \subset \Omega} m(B) & \forall A \subseteq \Omega \\
P l(A)=\sum_{A \cap B \neq \varphi} m(B) & \forall A \subseteq \Omega
\end{aligned}
$$

$\operatorname{Bel}(\mathrm{A})$ is the degree to which the evidence supports $\mathrm{A}$, and $\mathrm{Pl}(\mathrm{A})$ is the maximal degree of support that could be assigned to $A$, if there were more available evidence. $\mathrm{Pl}(\mathrm{A})$ may also be defined as the extent to which we fail to disbelieve the hypothesis of A.

\section{Evidential Networks in reliability analysis}

Evidential networks is a directed acyclic graph (DAG). An evidential network is a DAG $\boldsymbol{G}=((\boldsymbol{N}, \boldsymbol{A}), \boldsymbol{M})$ where $(N ; A)$ represents the graph, " $N$ " is a set of nodes, " $A$ " is a set of arcs and " $M$ " expresses the set of belief distributions that are distributed to each node. When a node is a root node, it's a priori belief mass table 
is defined. When a node is not a root node, its belief mass distribution is defined by a conditional belief mass table quantifying the relation between the nodes and its parents [16]. The network propagates basic belief assignments as an a priori belief mass on variables. A conditional belief function quantifies the dependency between a node and its parents. In the evidential networks using belief functions, the relations among the variables are usually represented by joint belief functions.

The idea is to assign a number between 0 and 1 indicating a degree of reliability belief, the term belief meaning that uncertainty quantification is mainly involved in the assessment of the degree of belief by experts with regard to uncertain information [8] such as $\Omega=[U p ;$ Down]:

$$
2^{\Omega}=[\varnothing] ;[U p] ;[\text { Down }] ;[U p ; \text { Down }]
$$

Where:

$[U p]: \quad$ component in the operating state,

[Down]: component in the failed state,

[Up; Down]: component in one of the two previous states, which describes an ignorance that represents epistemic uncertainty.

For an Evidential Networks, we develop by the same reasoning as probabilistic network, we associate to each component node of DBN model a conditional mass table CMT instead of CPT as we did in [1]. These CMT tables are based on parameters uncertainties $[\lambda \min ; \lambda \max ]$, leading to studying the three states of the components: Up; Down; Up; Down.

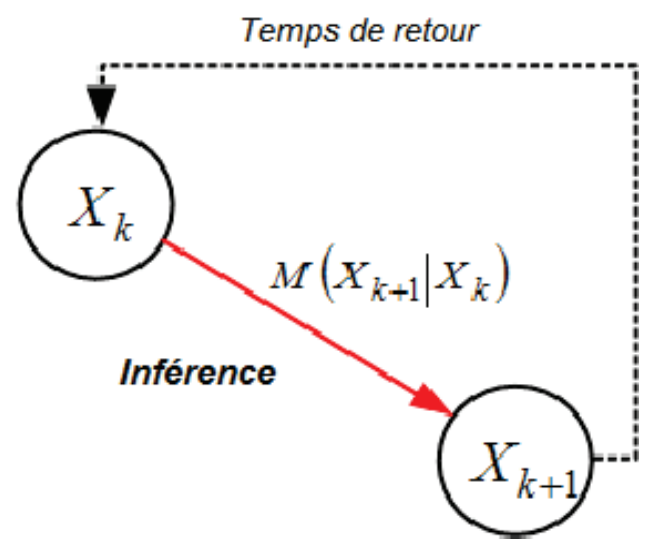

Fig. 3. Inference in belief network 


$$
M\left(X_{k+1} \mid X_{k}\right)
$$$$
=\left[\begin{array}{ccc}
m\left(\{U p\}^{k+1} \mid\{U p\}^{k}\right) & m\left(\{\text { Down }\}^{k+1} \mid\{U p\}^{k}\right) & m\left(\{U p, \text { Down }\}^{k+1} \mid\{U p\}^{k}\right) \\
m\left(\{U p\}^{k+1} \mid\{\text { Down }\}^{k}\right) & m\left(\{\text { Down }\}^{k+1} \mid\{\text { Down }\}^{k}\right) & m\left(\{\text { Up Down }\}^{k+1} \mid\{\text { Down }\}^{k}\right) \\
m\left(\{U p\}^{k+1} \mid\{U p, \text { Down }\}^{k}\right) & m\left(\{\text { Down }\}^{k+1} \mid\{\text { Up , Down }\}^{k}\right) & m\left(\{\text { Up Down }\}^{k+1} \mid\{U, \text { Down }\}^{k}\right)
\end{array}\right]
$$

$$
\mathrm{M}\left(\mathrm{X}_{\mathrm{k}+1} \mid \mathrm{X}_{\mathrm{k}}\right)=\left[\begin{array}{ccc}
1-\lambda \max & \lambda \min & \lambda \max -\lambda \min \\
0 & 1 & 0 \\
0 & \lambda \min & 1-\lambda \min
\end{array}\right]
$$

Such as:

$\boldsymbol{m}\left(\boldsymbol{D o w n} \boldsymbol{n}_{k+1} / \boldsymbol{U p}_{k}\right)=\lambda_{\text {max }}$ : The transition belief mass corresponds to the minimum of belief that the component falls down (it is sure that the component is down after the transition time).

$\boldsymbol{m}\left((\boldsymbol{U p} ; \boldsymbol{D o w n})_{k+1}=\boldsymbol{U} \boldsymbol{p}_{\boldsymbol{k}}\right)=\lambda_{\max }-\lambda_{\min }$ The transition mass corresponds to the uncertainty of the component hypothesis after the transition time. There is an ambiguity between the hypotheses Up and Down.

$\boldsymbol{m}\left(\boldsymbol{U} \boldsymbol{p}_{\boldsymbol{k}+1} / \boldsymbol{U} \boldsymbol{p}_{\boldsymbol{k}}\right)=1-\lambda_{\text {min }}$ : The transition mass corresponds to the belief mass transition that it is sure that there is no failure.

$\boldsymbol{m}\left(\boldsymbol{D o w n}_{k+1} /\left(\boldsymbol{U p} ; \boldsymbol{D o w n}_{k}\right)=\lambda_{\min }\right.$ : This belief transition mass allocated to Down corresponds to the minimum of belief that the component fails down.

$\boldsymbol{m}\left(\boldsymbol{U} \boldsymbol{p}_{\boldsymbol{k}+1} /(\boldsymbol{U p} ; \boldsymbol{D o w n})_{\boldsymbol{k}}\right)=\mathbf{0}$ : The following equation for temporal nodes, the temporal conditional mass table of each component is computed.

\section{Uncertainties related to multi-domain interaction}

Our goal is to adapt this Evidential Network using belief network to the method proposed in $[19,20]$ by taking into account the interactive aspect of mechatronic systems during the reliability analysis. Also, we consider the epistemic uncertainties related to the treated multi-domain interaction. 


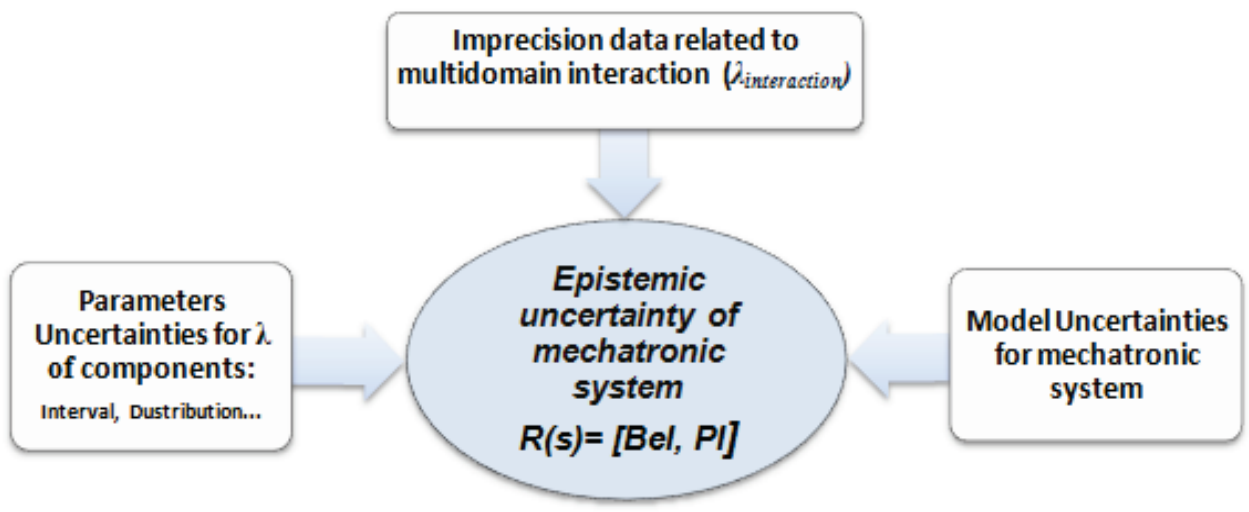

Fig. 4. Reliability model for mechatronic system with uncertainties

Multi-domain interaction is a cause and effect relationship for a component failure mechanism, which comes from other fields as presented in fig. 6, because this interaction, failure rate of influenced component, is changed. For example, a multi-domain interaction: temperature, humidity, overpressure, electro-migration et cetera. Each interaction is affected by a stress law to model it, but to represent the uncertainties related to multi-domain interaction, we have to frame the failure rate of the influenced component as demonstrated in tab. 1. Thereafter, we create a new table (tab. 2) of a conditional belief mass associated to the new link representing the interaction, using the same logic as in the failure rates matrix, by adding parameters related to interaction.

Table 1

Failure rate uncertainties of Multi-domain interaction

\begin{tabular}{|l|l|l|l||}
\hline \multicolumn{1}{|c|}{$\begin{array}{c}\text { Multi-domain } \\
\text { Interaction }\end{array}$} & \multicolumn{1}{|c|}{ Stress law } & \multicolumn{1}{|c||}{$\lambda_{\text {Interaction-min }}$} & \multicolumn{1}{|c||}{$\lambda_{\text {Interaction-max }}$} \\
\hline Temperature & Arrhenius Law & $\lambda_{\text {Arrhenius-min }}$ & $\lambda_{\text {Arrhenius-max }}$ \\
Humidity & Peck law & $\lambda_{\text {Peck-min }}$ & $\lambda_{\text {Peck-max }}$ \\
Electro-migration & Black law & $\lambda_{\text {Black-min }}$ & $\lambda_{\text {Black-max }}$ \\
Over pressure & Power inverse law & $\lambda_{\text {Power inverse-min }}$ & $\lambda_{\text {Power inverse-max }}$ \\
Multiple interactions & Cox law & $\lambda_{\text {Cox-min }}$ & $\lambda_{\text {Cox-max }}$ \\
\hline \hline
\end{tabular}

In Evidential Networks, the Conditional Mass Table (CMT) is established with the same reasoning as the last matrix of uncertainties, and for each conditional mass associated to the states Up; Down; Up-Down, we specify a multi-domain 
interaction $\left[\lambda_{\text {Interaction-min }} ; \lambda_{\text {Interaction-max }}\right]$ as interval of uncertainty calculated depending on the stress law selected in first part.

\section{Table 2}

Conditional Mass table of influenced Node

\begin{tabular}{|c|c|c|c|c|}
\hline $\mathrm{X}$ & $\mathrm{Y}$ & Up & Down & Up, Down \\
\hline Up & $\begin{array}{l}\text { Up } \\
\text { Down } \\
\text { Up-Down }\end{array}$ & $\begin{array}{l}1-\lambda_{\text {refmax }} \\
0 \\
0\end{array}$ & $\begin{array}{l}\lambda_{\text {ref-min }} \\
1 \\
\lambda_{\text {ref-min }}\end{array}$ & $\begin{array}{l}\lambda_{\text {ref-max }}-\lambda_{\text {ref-min }} \\
0 \\
1-\lambda_{\text {ref-min }}\end{array}$ \\
\hline Down & $\begin{array}{l}\text { Up } \\
\text { Down } \\
\text { Up-Down }\end{array}$ & $\begin{array}{l}1-\lambda \text { Interaction-max } \\
0 \\
0\end{array}$ & $\begin{array}{l}\lambda_{\text {Interaction-min }} \\
1 \\
1-\lambda_{\text {Interaction-min }}\end{array}$ & $\begin{array}{l}\lambda_{\text {Interaction-max }}-\lambda_{\text {Interaction-min }} \\
0 \\
1-\lambda_{\text {Interaction-min }}\end{array}$ \\
\hline $\begin{array}{l}\text { Up- } \\
\text { Down }\end{array}$ & $\begin{array}{l}\text { Up } \\
\text { Down } \\
\text { Up-Down }\end{array}$ & $\begin{array}{l}1-\lambda_{\text {Interaction-max }} \\
0 \\
0\end{array}$ & $\begin{array}{l}1-\lambda \text { Interaction-min } \\
1 \\
0\end{array}$ & $\begin{array}{l}\lambda_{\text {Interaction-max }}-\lambda_{\text {Interaction-min }} \\
0 \\
1\end{array}$ \\
\hline
\end{tabular}

To represent the belief functions Pls and Bel, we created additional nodes P1 and $\mathrm{B} 1$, related to the mass tables 3 and 4 to have information on probability boxes that bound the real value of the failure probability for the mechatronic system. If no epistemic uncertainty is introduced in component states $\boldsymbol{m}(\boldsymbol{U p}$ or Down $)=\mathbf{0}$, then the previously computed reliability value is confirmed. When $\boldsymbol{B e l}$ and $\boldsymbol{P l}$ measures are not equals, the basic belief assignment on the system state expresses an epistemic uncertainty bound the system reliability, a further decision can be made according to this uncertainty. Using these calculus rules of reliability $R s$, we obtain an interesting characteristic that is the bounding of the probability by the measures of belief and plausibility:

$$
\operatorname{Bel}(\mathrm{A}) \leq \mathrm{RS}(\mathrm{A}) \leq \mathrm{P} \text { ls(A) }
$$

\section{Application on Smart Actuator}

To illustrate the proposed method for the estimation of the predicted reliability of the mechatronic system, we chose an application of PACK'AERO [19, 21], the Smart Actuator. This system is a mechatronic system designed to carry out the unloading function of the wagons. The main issue is a multi-domain interaction 
coil/bearing as an interaction between the electric domain and the mechanical domain.

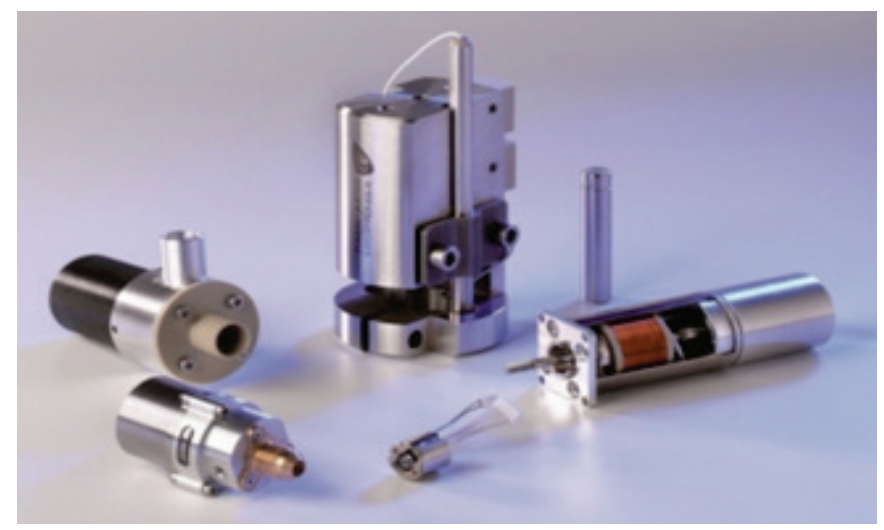

Fig. 5. Smart actuator system

The application revealed some critical elements: Control and diagnostic card of the actuator, Hall sensor, coil and guide bearing. A series configuration was considered, and the rest of the elements of the system are not taken into account. Figure 5 presents the actuator system.

\subsection{Uncertainties of Actuator system parameters}

To argue the interest of our second contribution, we consider the uncertainty introduced in the state of the components only related to the multi-domain interaction: Guide bearing and electrical coil. An imprecision concerning the failure rate of each component is given as an interval $\left[\lambda_{\min } ; \lambda_{\max }\right]$, with a confidence level of $90 \%$ of data presented in tab. 3 .

Table 3

\section{Component failure rate with uncertainties}

\begin{tabular}{||l|l|l||}
\hline \multicolumn{1}{|c|}{ Component } & \multicolumn{1}{|c||}{ Failure rate $\min (1 / \mathrm{h})$} & \multicolumn{1}{|c|}{ Failure rate max $(1 / \mathrm{h})$} \\
\hline Coil Electrical & $1.16 \mathrm{E}-06$ & $1.66 \mathrm{E}-06$ \\
Hall sensor & $1.21 \mathrm{E}-07$ & $1.7 \mathrm{E}-07$ \\
Control Card & $1.72 \mathrm{E}-07$ & $2.22 \mathrm{E}-07$ \\
Guide Bearing & $\mathrm{MTTF}=0.81 \mathrm{E}+03$ & $\mathrm{MTTF}=1.3 \mathrm{E}+04$ \\
& $\beta=1.5$ & $\beta=1.5$ \\
Card of Condit. & $1.16 \mathrm{E}-06$ & $1.66 \mathrm{E}-06$ \\
Inductor & $5.10 \mathrm{E}-05$ & $5.60 \mathrm{E}-05$ \\
\hline
\end{tabular}




\subsection{Model Uncertainties of Actuator System}

The components are considered to be in series and the CMT related to the logic node (ET) with the epistemic uncertainty, which is presented in tab. 4:

Table 4

\section{Conditional Mass table of system Node}

\begin{tabular}{||l|l|l|l|l||}
\hline \multicolumn{1}{|c|}{ Bear $\mathrm{t}+1$} & \multicolumn{1}{|c|}{ Coil $\mathrm{t}+1$} & Up & Down & Up-Down \\
\hline Up & Up & 1 & 0 & 0 \\
Down & Down & 0 & 1 & 0 \\
Up-Down & Up-Down & 0 & 0 & 1 \\
Up & Up & 0 & 1 & 0 \\
Down & Down & 0 & 1 & 0 \\
Up-Down & Up-Down & 0 & 1 & 0 \\
Up & Up & 0 & 0 & 1 \\
Down & Down & 0 & 1 & 0 \\
Up-Down & Up-Down & 0 & 0 & 1 \\
\hline \hline
\end{tabular}

The simulation of this Evidential network is done using the BAYESIA LAB V.06 software as presented in fig. 6 .
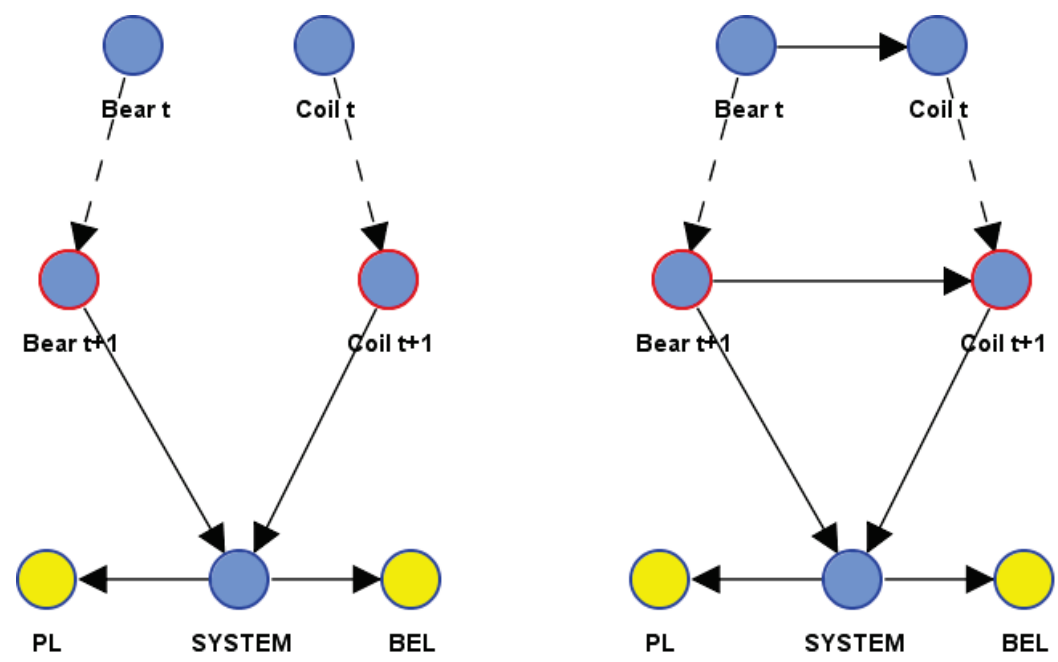

Fig. 6. Evidential network for actuator system with/without interaction 
For the other components, based on calculations of conditional masses presented in tables 5 and 6 .

Table 5

\section{CMT of BEL node}

\begin{tabular}{|l|c|c||}
\hline \multicolumn{1}{|c|}{ System } & Plausibility & Not Plausibility \\
\hline Up & 1 & 0 \\
\hline Down & 0 & 1 \\
\hline Up-Down & 0 & 1 \\
\hline
\end{tabular}

Table 6

CMT of PL node

\begin{tabular}{|l|c|c|}
\hline \multicolumn{1}{|c|}{ System } & Belief & Not Belief \\
\hline Up & 1 & 0 \\
\hline Down & 0 & 1 \\
\hline Up-Down & 1 & 0 \\
\hline
\end{tabular}

\subsection{Uncertainty related to the interaction Coil/Bear}

The multi-domain interaction treated in this application is between Electrical Coil/ Mechanical Bear. This interaction is modelled by Arrhenius law as influence factor, so the uncertainty is presented as following interval $\left[\lambda_{\text {Interaction-min}} ; \lambda_{\text {Interaction- }}\right.$ $\left.{ }_{\max }\right]=\left[\lambda_{\text {Arrh-min }} ; \lambda_{\text {Arrh-max }}\right]$ as presented in tab. 7.

Table 7

Conditional Mass table of coil" node $t+1$

\begin{tabular}{|l|l|l|l|l|}
\hline Bear & \multicolumn{1}{|c|}{ Coil } & \multicolumn{1}{c|}{ Up } & \multicolumn{1}{c|}{ Down } & \multicolumn{1}{c|}{ Up, Down } \\
\hline \multirow{3}{*}{ Up } & Up & $1-\lambda_{\text {refmax }}$ & $\lambda_{\text {ref-min }}$ & $\lambda_{\text {ref-max }}-\lambda_{\text {refmin }}$ \\
& Down & 0 & 1 & 0 \\
\hline \multirow{3}{*}{ Down } & Up-Down & 0 & $\lambda_{\text {ref-min }}$ & $1-\lambda_{\text {ref-min }}$ \\
& Up & $1-\lambda_{\text {Arrh-max }}$ & $\lambda_{\text {Arrh-min }}$ & $\lambda_{\text {Arrh-max }}-\lambda_{\text {Arrh-min }}$ \\
& Down & 0 & 1 & 0 \\
& Up-own & 0 & $1-\lambda_{\text {Arrh-min }}$ & $1-\lambda_{\text {Arrh-min }}$ \\
\hline \hline
\end{tabular}




\begin{tabular}{|l|l|l|l|l||}
\hline \multirow{3}{*}{ Up-Down } & Up & $1-\lambda_{\text {Arrh-max }}$ & $1-\lambda_{\text {Arrh-min }}$ & $\lambda_{\text {Arrh-max }}-\lambda_{\text {Arrh-min }}$ \\
& Down & 0 & 1 & 0 \\
& Up-Down & 0 & 0 & 1 \\
\hline
\end{tabular}

\subsection{Simulation in BNT MATLAB Toolbox}

To model the whole system by considering the six critical elements: Control and diagnostic card of the actuator, Hall sensor, coil and guide bearing, we use the BNT toolbox in the Matlab. By generating and executing the code of our evidential network, an EN graph of smart actuator is presented in fig. 7.

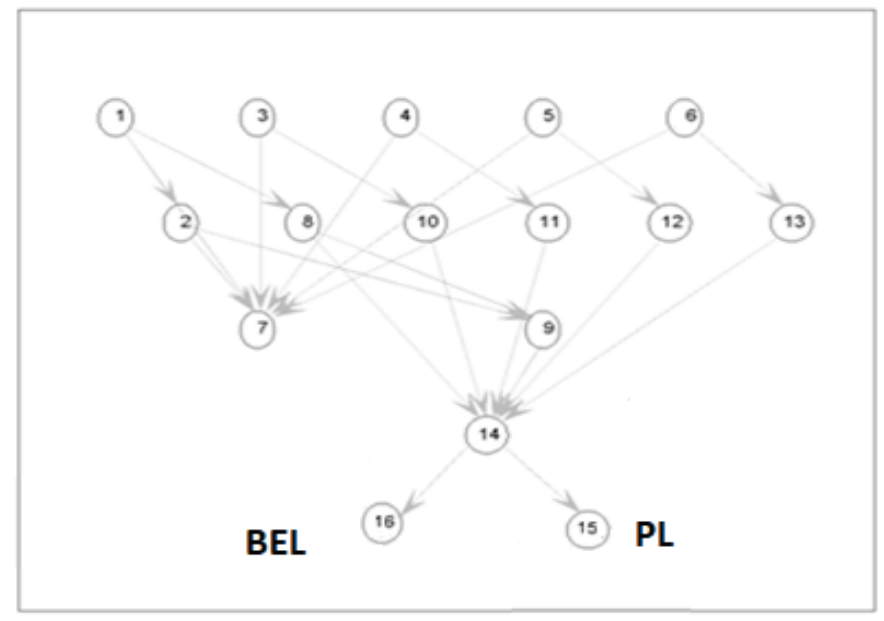

Fig. 7. Evidential network for actuator system with/without interaction

\subsection{Results}

The measures of belief and plausibility functions of the actuator system are indicated in fig. 8 by representing the two cases with/without Multi-domain interactions. The simulation done for over 5000 hours (about 7 months) by taking into account the interaction, shows that smart actuator reliability is in the range of $[\mathbf{0 . 3 1 3} ; \mathbf{0 . 3 3 4}]$. This interval shows the worst value of the reliability of the system. $\mathbf{m}[(\mathbf{U p}$, Down $) \mathbf{t}=\mathbf{5 0 0 0}]=\mathbf{0 , 0 2 1}$ represents the epistemic uncertainty of reliability of the smart actuator without taking into account the interaction Bear/Coil. 


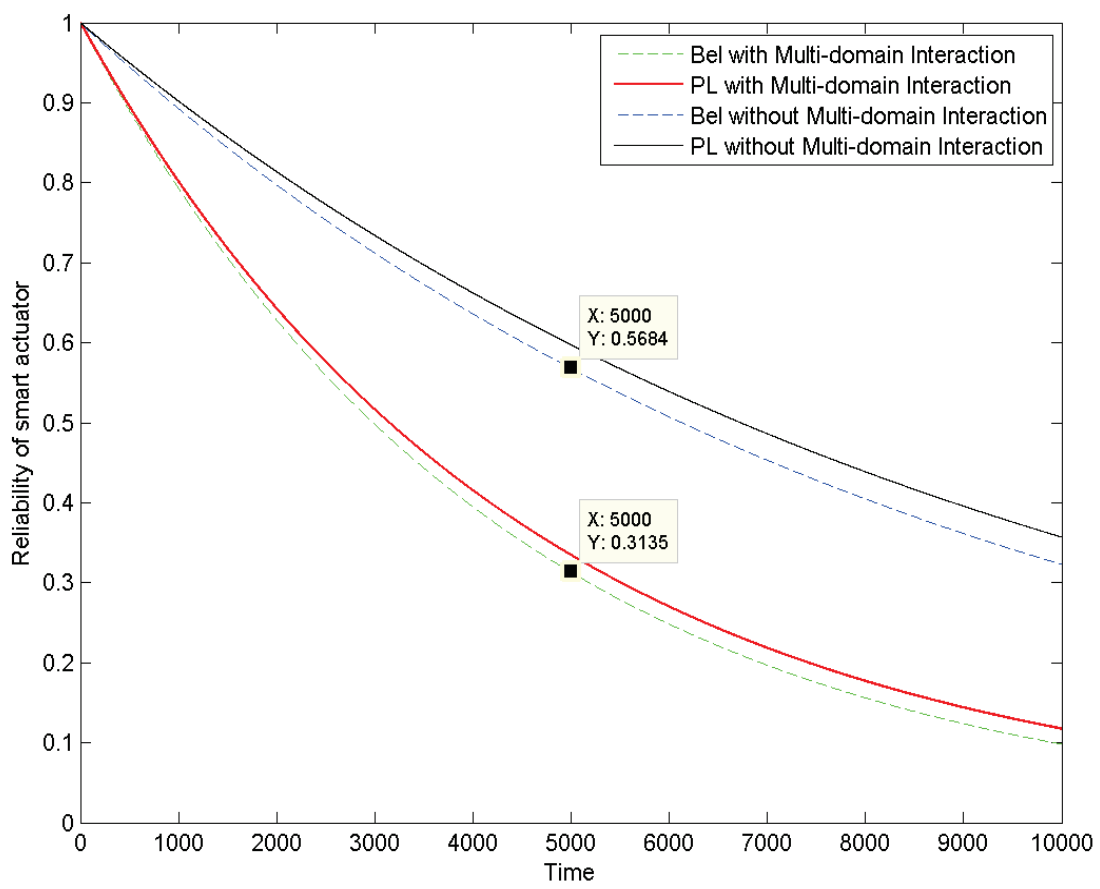

Fig. 8. Reliability of mechatronic system under uncertainties

In the second case (without interaction), the belief network of the actuator is bounded by the interval $[\mathbf{0 . 5 6 8 ;} \mathbf{0 . 5 9 7}]$ as in fig. 8 . Also, this simulation shows the propagation of uncertainties from each component to the whole system. Finally, the epistemic uncertainty is illustrated by the interval: $\mathbf{m}[(\mathbf{U p}$, Down $) \mathbf{t}=\mathbf{5 0 0 0}]=\mathbf{0 , 0 2 9}$ represents the epistemic uncertainty of reliability of the smart actuator by taking into account the interaction Bear/Coil. The final results are presented in tab. 8 .

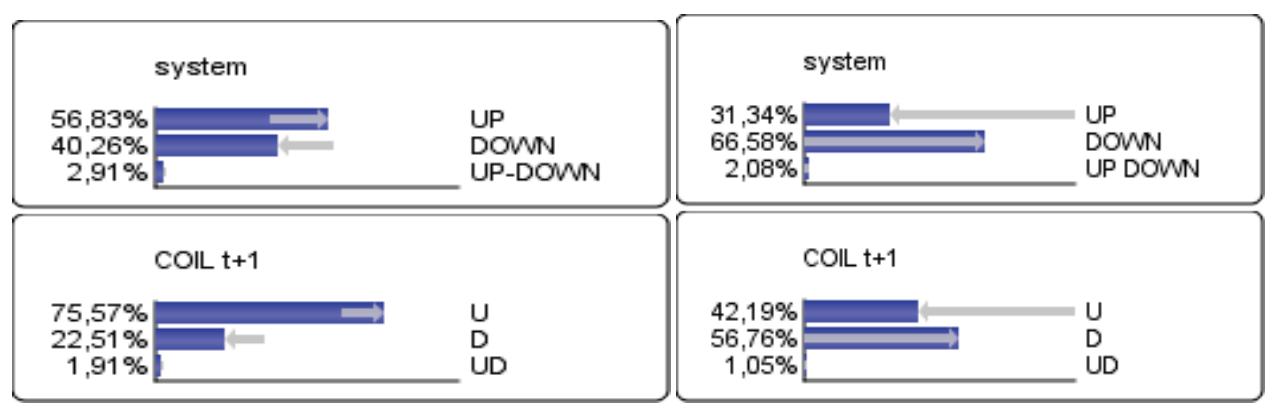

Fig. 9. Pl and Bel measures on Electrical coil and system 


\section{Table 8}

Comparison of the Reliability of system

\begin{tabular}{|l|l|l|}
\hline & \multicolumn{1}{|c|}{ BEL Function } & \multicolumn{1}{|c|}{ PL Function } \\
\hline Without Interaction Coil/Bear & 0.568 & 0.597 \\
\hline With Interaction Coil/ Bear & 0.313 & 0.334 \\
\hline
\end{tabular}

\section{Conclusion}

The use of the belief and plausibility measures can give the engineers more credible information and allows computing the worst value of the reliability of the system. The coupling between the DBN and the belief functions is a powerful method allowing to predict the reliability of mechatronic systems by taking into account the epistemic uncertainties, as well as the propagation of uncertainties related to the multi-domain interaction.

This methodology allows analyzing both the uncertainties and the multidomains interactions, within this framework, reliability of mechatronic system is no longer represented by a precise value of probability, but with an interval [Bel, $\mathrm{Pl}$ ]; that bounds an uncertain measure about the reliability. Gathering more data tightens up the interval until it converges to a precise value of reliability. Also, it makes it possible to easily model the epistemic uncertainties without having to choose a probability density function (normal, normal log, triangular, et cetera) as we did in modelling reliability by using Petri Network [20]. Our methodology can help engineers to detect with precision the influence of multi-domain interactions in system reliability and lead designers to search for new technological solutions. As at $\mathrm{t}=5000 \mathrm{~h}$ for the two cases perspectives, the study of a reconfigurable aspect of mechatronic systems seems an interesting way for future studies.

\section{References}

1. Oberkampf, W.L., Helton J.C., Joslyn C.A., Wojtkiewicz S.F., Ferson S.: Challenge Problems: Uncertainty in System Response Given Uncertain Parameters, Reliability Engineering and System Safety, vol. 85, no. 1-3, 2004.

2. Utkin L.V., Coolen F.: Imprecise reliability: An introductory overview. In: Intelligence in Reliability Engineering. Ed. by G. Levitin. Springer Berlin Heidelberg, 2007. 
3. Coit D., Jin T., Wattanapong Sakorn N.: System optimization with Component Reliability estimation uncertainty: A multi-criteria Approach, IEEE Transactions on Reliability, 53(2004).

4. Sallak M., Schon W., Felipe A.: The Transferable Belief Model for reliability analysis of systems with data uncertainties and failure dependencies. Proceedings of the Institution of Mechanical Engineers, Part O: Journal of Risk and Reliability, SAGE Publications.224,4, 2010.

5. Tu Duong D., Vasseur TD., Dieulle D.: Representation of parameter uncertainty with evidence theory in Probabilistic Risk Assessment. In Proceeding of the workshop on the theory of belief function, France, Brest, 2010.

6. Simon C., Weber Ph., Evsuko A.: Bayesian networks inference algorithm to implementDempster Shafer theory in reliability analysis. Reliability Engineering and System Safety, elsevier.93, 2008.

7. Weber Ph., Simon C.: Imprecise reliability by evidential networks. Proceedings of the Institution of Mechanical Engineers Part, Journal of Risk and Reliability, SAGE Publications, 223, 2009.

8. Honda K., Washizaki H., Fukazawa Y.: Generalized Software Reliability Model Considering Uncertainty and Dynamics: Model and Applications. International Journal of Software Engineering. World scientific Publishing. 27, 2017.

9. Felipe Aguirre et al.: Application of Evidential Networks in quantitative analysis of rail way accidents. Journal of Risk and Reliability - Special issue, 2013.

10. Kochs H.D., Kongniratsaikul P.: Comparing System Reliability Considering Insufficient Knowledge: Application to HVDC Converter Stations, Proceedings of IEEE Power Energy Society General Meeting (IEEE PES GM), July 2012, San Diego, CA USA, 2012.

11. Heard A., Pensky M.: Confidence Intervals for Reliability and Quantile Functions With Application to NASA Space Flight Data, IEEE Transactions on Reliability, Volume 55, Issue 4, Dec. 2006.

12. Kongniratsaikul P.: Evaluation of System Reliability Using the Example of HVDC ystems, Master Thesis, University of Duisburg-Essen, Germany, 2009.

13. Simon Ch., Weber P.: Evidential Networks for Reliability Analysis and Performance Evaluation of Systems With Imprecise Knowledge. In: IEEE Transactions on Reliability 58.1, Mar. 2009.

14. Glenn Shafer. Perspectives on the theory and practice of belief functions. International Journal of Approximate Reasoning.3, 1990.

15. Limbourg Ph., Savic R., Petersen J., Kochs H.-D.: Modelling uncertainty in fault tree analyses using evidence theory. Proceedings of the Institution of Mechanical Engineers, Part O: Journal of Risk and Reliability 222.3 Sept. 2008.

16. Le Duy, Tu Duong: Traitement des incertitudes dans les applications des Etudes Probabilistes de Surete Nucleaire. PhD thesis. Troyes, France: University of Technology of Troyes, Dec. 2011. 
17. Xiaomin You, Tonon F.: Event-tree analysis with imprecise probabilities. In: Risk analysis: an official publication of the Society for Risk Analysis 32.2.

18. Zhong X., Ichchou M., Saidi A.: Reliability assessment of complex mechatronic systems using a modified nonparametric belief propagation algorithm. Reliability Engineering and System Safety; 2010, DOI:10.1016/j.ress.2010.05.004.

19. Amrani N.B., Saintis L., Sarsri D., Barreau M.: Bayesian Reliability Model for mechatronic system by taking into account the multi-domain interaction. Journal of KONBIN, Vol. 49, 2019, DOI 10.2478/jok-2019-0002.

20. Amrani N.B., Saintis L., Sarsri D., Barreau M.: Prise en compte des interactions multidomaines lors de l'évaluation de la fiabilité prévisionnelle des systèmes mécatroniques. Proc. Int. Lambda-mu20. France, Saint Malo, 2016.

21. Habchi G., Barthod C.: An overall methodology for reliability prediction of mechatronic systems design with industrial application. Reliability Engineering and System Safety.155, 2016. 